\title{
Lion/Lioness Gender Recognition
}

\author{
Hari Chandana Karnam Rajendraprasad \\ Graduate Student, Dept. of Computer Engineering, \\ California State University, Sacramento, California, United States
}

\begin{abstract}
A Lion's facial structure provides important features about its gender. This project presents an approach for gender detection from images considering facial structure as a region of interest. At the end of the project the system should be able to automatically detect the gender of a lion based on collected data. The project can potentially be expanded to cover a wider range of animals that can be used to keep record and prevent the extinction of protected species.
\end{abstract}

\section{General Terms}

Machine Vision, Classification problem, image processing

\section{Keywords}

Animal, face, lion, lioness, recognition, gender, specie

\section{INTRODUCTION}

Modern software and hardware tools have allowed for advanced technique in facial recognition. What was formerly seen as science fiction only decades ago, are now realized. Today, there are some advanced facial recognition software that allows to compare and even track a person in real-time. These types of tools consider a facial structure and any type of elements that are unique and uses that database as query to track and identify similar faces. In this project, similar type of algorithm is used to identify a lion, whether it's a male or female lion based on its unique facial feature.

There are some basic characteristics that allows us to distinguish between the male lion and its female counterpart. The scope of this project is limited to lions that have already aged. A male lion, in full age has a great amount of mane (facial hair) in comparison to a female lion. This feature can be used to differentiate them. A child to differentiate between the two genders of lions by showing a series of male and female lions and teach them based on difference between their features. Similarly, the program will be trained to identify and differentiate between a male and female lion. Two datasets with a series of male lions and female lions are used for comparison.

\subsection{Creating the datasets}

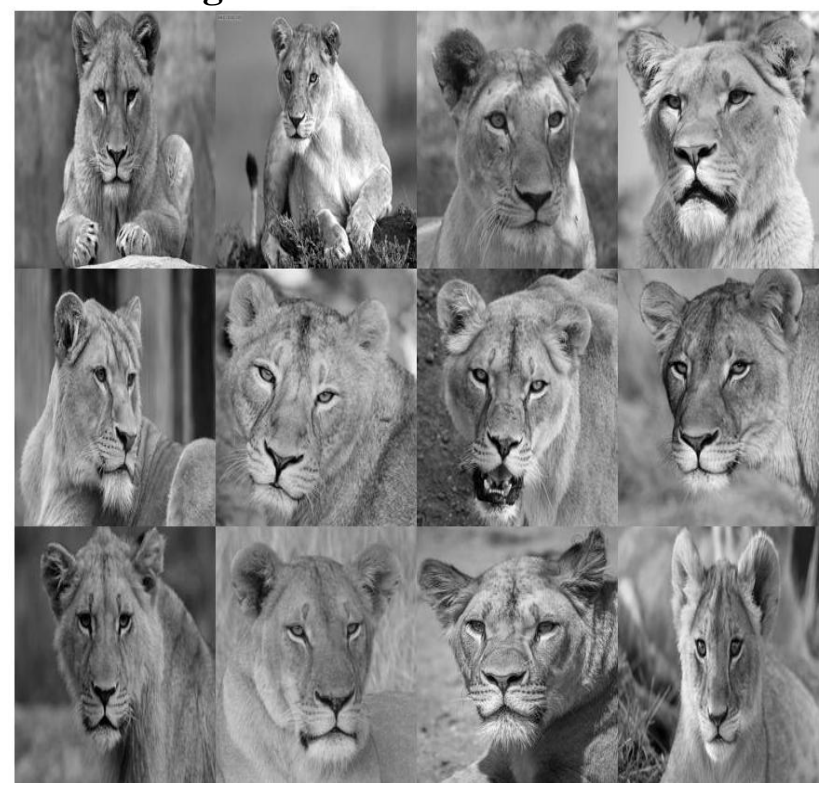

Figure 1. Female Lion

In the project file two subdirectories are created, each containing a set of images of 12 male lions and 12 female lions in the dataset accordingly. All the images are converted into grayscale. And each image is resized to a 500x500 pixel image. This will serve as for our initial testing of our algorithm. See figure 1 and 2 for a montage of male and female lions.

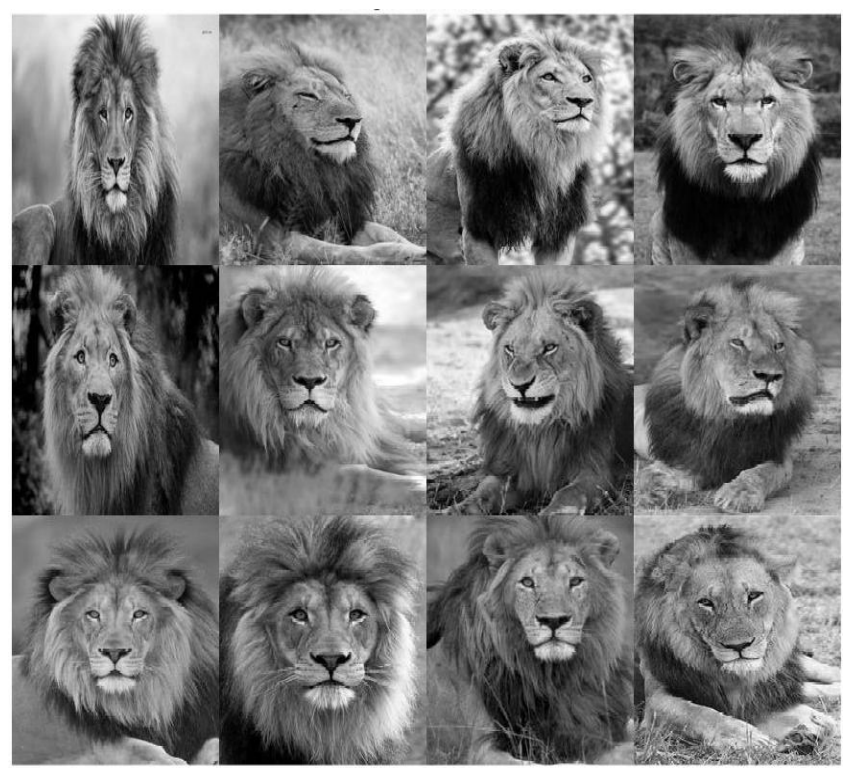

Figure 2. Male Lion 


\subsection{Extraction of HoG Features}

The algorithm is based on the method provided by MATLAB which is implemented for facial recognition on people. The histogram of oriented gradients (HOG) is a feature descriptor used in computer vision and image processing for object detection. The technique counts occurrences of gradient orientation in localized portions of an image. ${ }^{[4]}$

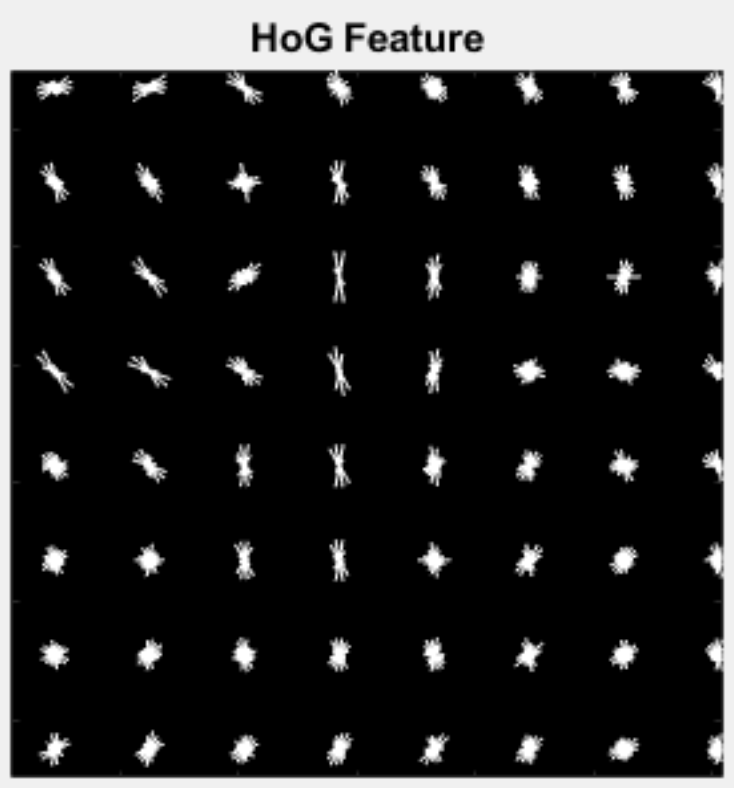

Figure 3. Extracted HoG Features from an image.

\section{PROCEDURE}

This method is similar to that of edge orientation histograms, scale invariant feature transform descriptors, and shape contexts, but differs in that it is computed on a dense grid of uniformly spaced cells and uses overlapping local contrast normalization for improved accuracy ${ }^{[4]}$. HoG feature greatly differs between a male lion and a female lion because of mane. Figures 4 and 5 shows the extracted HoG features.

Data set is split into two as training and testing in 80:20 ratio. So, training set comprises of 20 images and testing set comprises of 4 images. HoG features are extracted for each image in data set and stored as training features. extractHOGFeatures () is the built-in function used to extract the HoG features. The returned features encode local shape information from regions within an image.

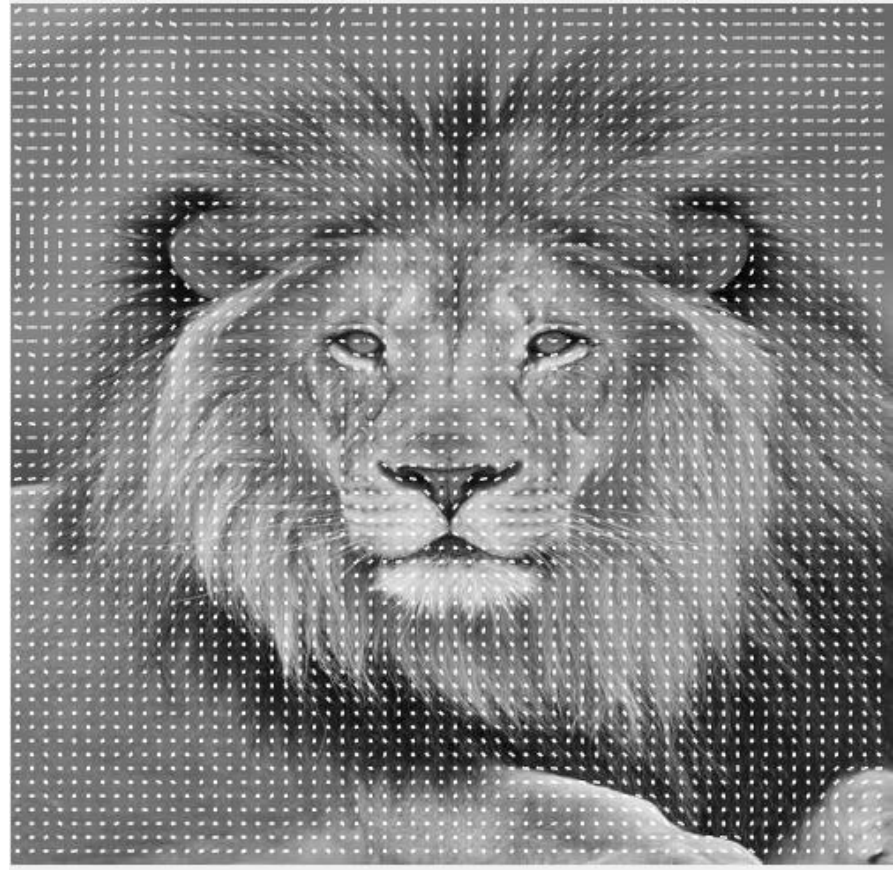

Figure.4 Extracted HoG Feature of male lion

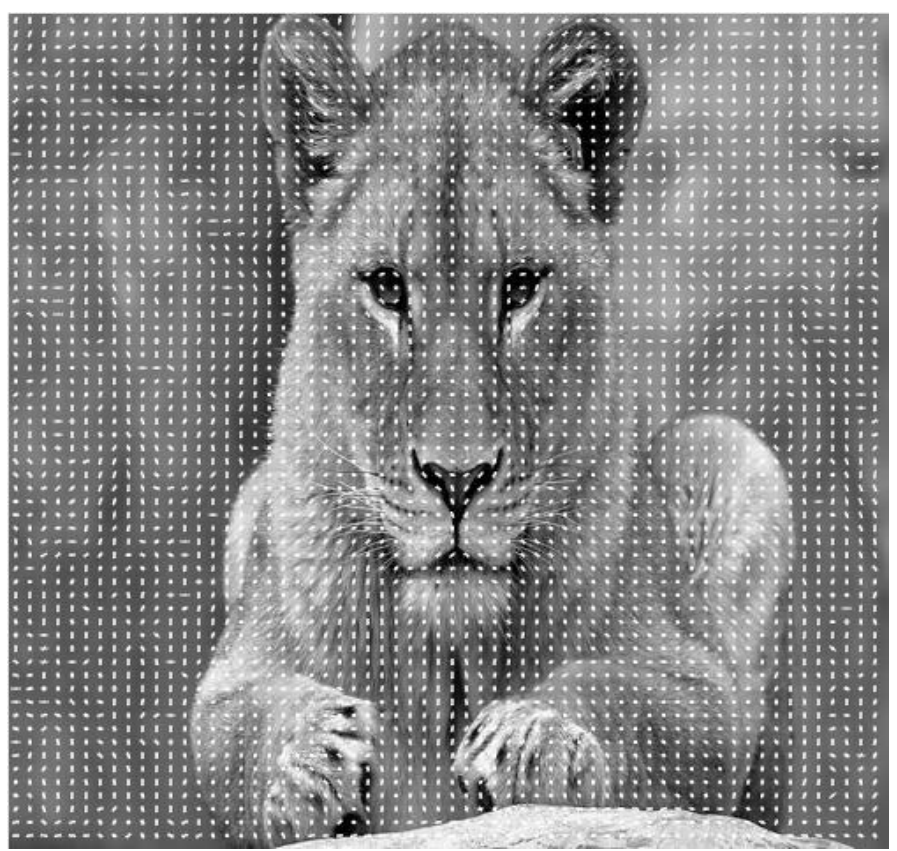

Figure 5. Extracted HoG feature of female lion

The features of the two classes are classified using a built-in function by MATLAB fitcecoc and stored in face classifier. fitcecoc(Tbl,ResponseVarName) returns a full, trained, multiclass, error-correcting output codes (ECOC) model using the predictors in table $\mathrm{Tbl}$ and the class labels in Tbl.ResponseVarName. fitcecoc uses $\mathrm{K}(\mathrm{K}-1) / 2$ binary support vector machine (SVM) models using the one-versus-one coding design, where $\mathrm{K}$ is the number of unique class labels (levels). ${ }^{[5,6,7]}$ Similarly the HoG features are extracted for the testing data and later used as query to the built-in function predict ()$^{[8]}$. predict $(\mathrm{Mdl}, \mathrm{X})$ returns a vector of predicted class labels (label) for the predictor data in the table or matrix $\mathrm{X}$, based on the trained multiclass error-correcting output codes (ECOC) model. ${ }^{[9,10]}$ 


\section{RESULTS}

Two images from each data set is used to test the program and results are accurate. If the extracted $\mathrm{HoG}$ features matches the respective class, it pulls the first image in the dataset as matched face. Figures 6 and 7 shows the results of testing set with a dataset of size 24 .
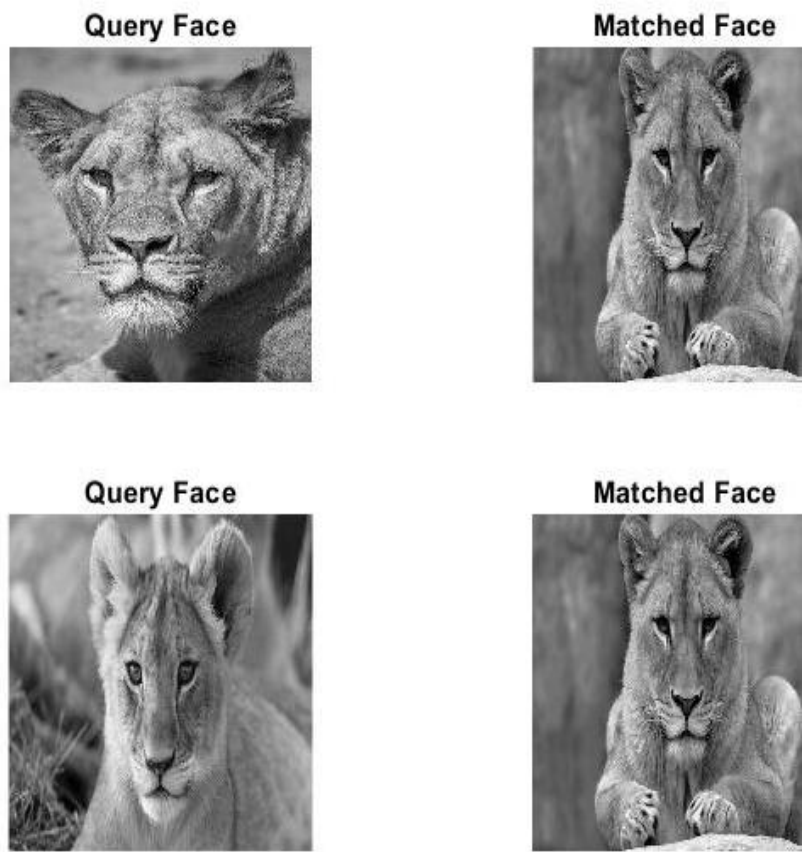

Figure 6. Query of Female Lion
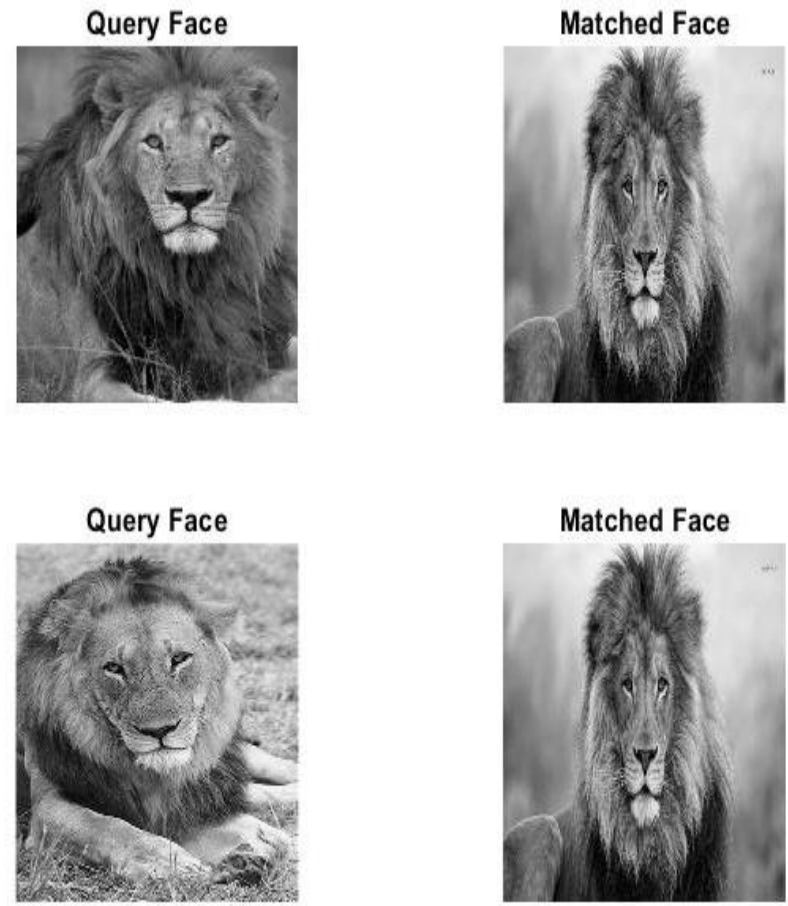

Figure 7. Query of Male Lion

\subsection{Increasing the dataset}

Dataset is increased to 40 images, 20 for the male and 20 for the female lions. Figure 8 and 9 shows a montage of the dataset with an increased size.

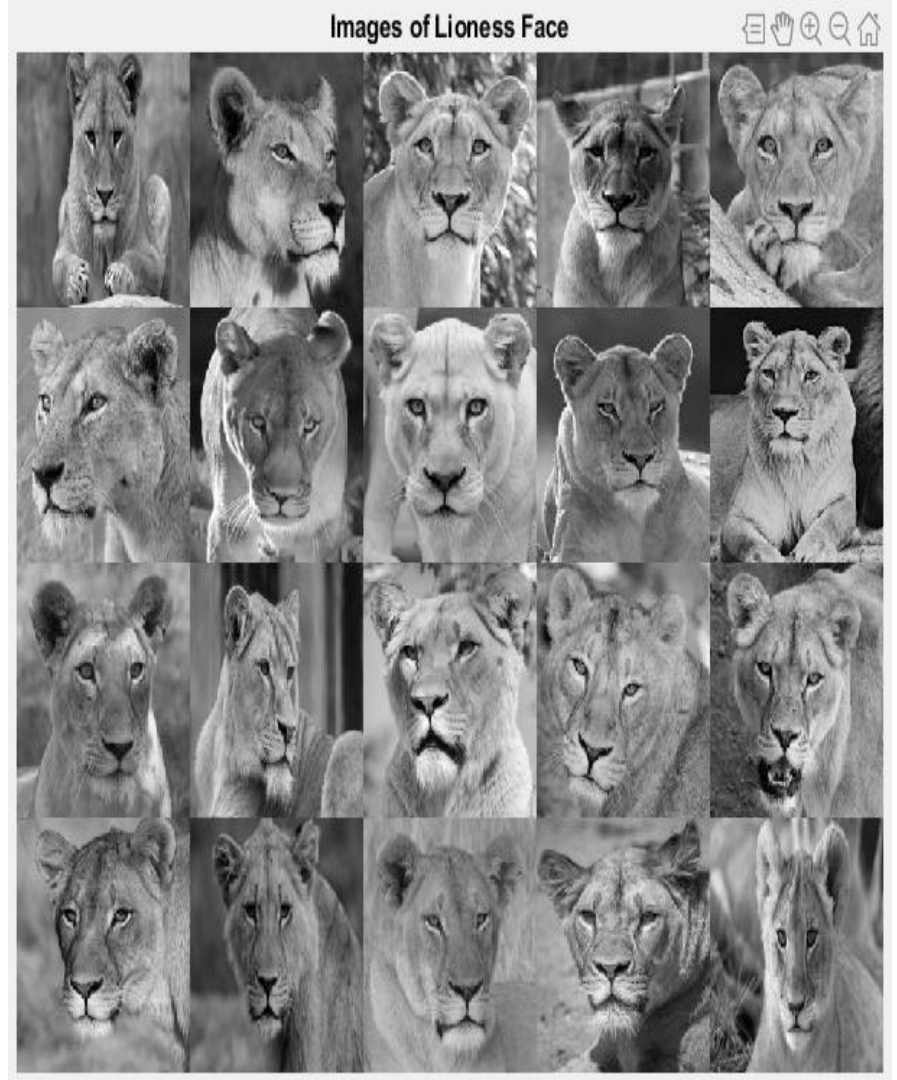

Figure 8. increased dataset of female lion

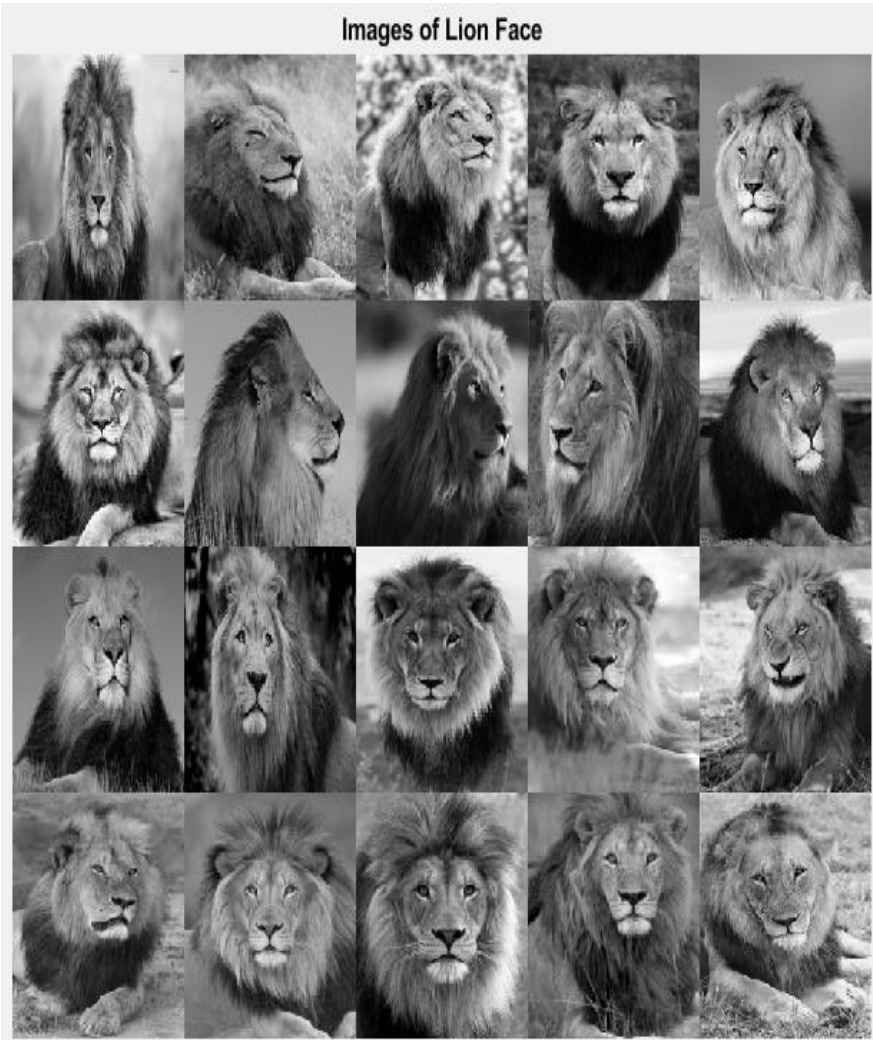

Figure 9. Dataset of Male Lion increase to 20 


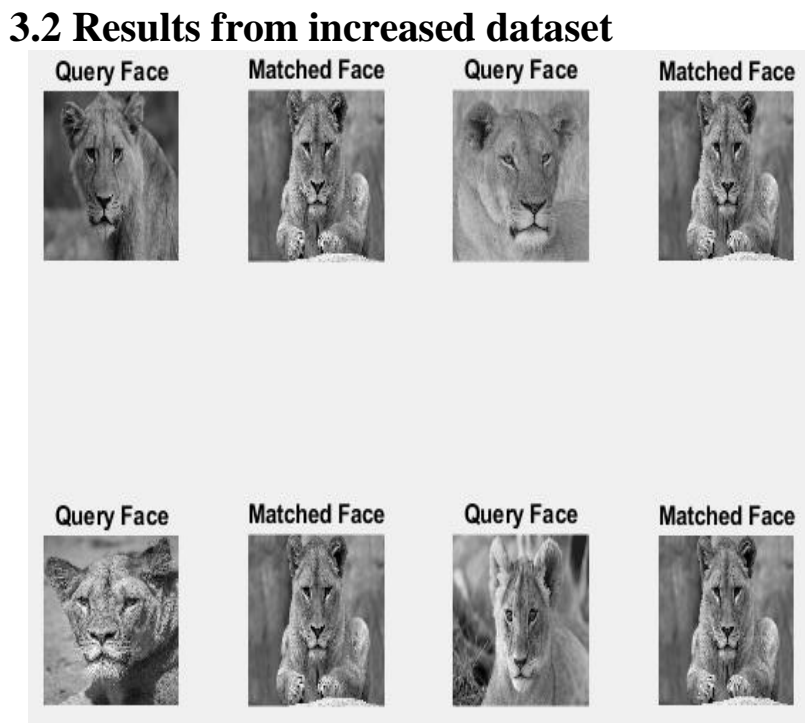

Figure 10. Query of Lioness

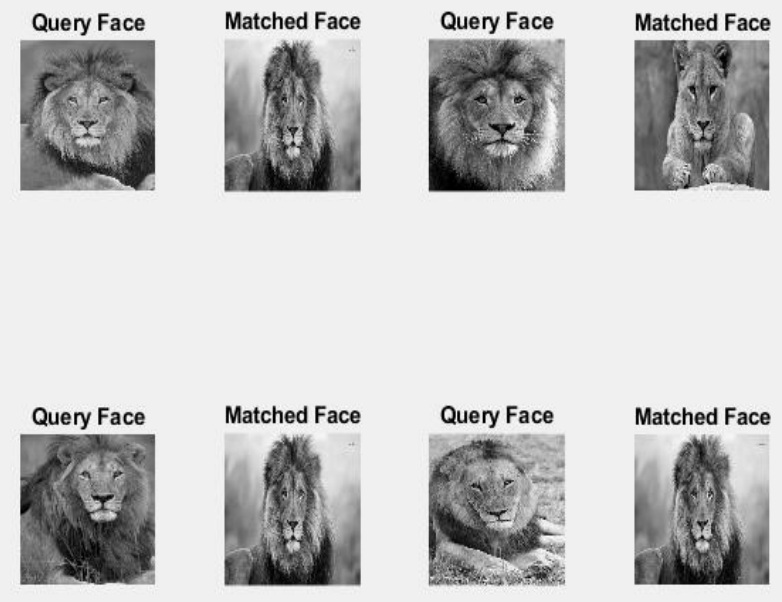

Figure 11. Query of Male Lions

\section{CONCLUSIONS}

In conclusion, face detection in animals through image processing is an important and interesting challenge. Its application can be broadening to cover a variety of subjects and solve an unspeakable amount of problems where our imagination is the limit. For example, this project can be used in conducting a survey of animals in a biosphere reserve for the protection of endangered species. According to the world wild life, there are currently 18 different type of species on the critically endangered list, and 29 on the endangered list ${ }^{[11]}$. Just by having a visual of the animal we can keep record of animals and determine what species are becoming endangered and adding them to the protected list as needed.

\section{REFERENCES}

[1] Emon Kumar Dey, Mohsin Khan, Md Haider Ali, "Computer Vision-Based Gender Detection from Facial Image," International Journal of Advanced Computer Science, Vol.3, No.8, Pp428-433, Aug. 2013.

[2] Dalal, N. and B. Triggs. "Histograms of Oriented Gradients for Human Detection", IEEE Computer Society Conference on Computer Vision and Pattern Recognition, Vol. 1 (June 2005), pp. 886-893.W.-K. Chen, Linear Networks and Systems (Book style). Belmont, CA: Wadsworth, 1993, pp. 123-135.

[3] Nehemiah, A. "Code for Face Recognition with Matlab Webinar", (November 2015). [Online]. Available: http://www.citethisforme.com/ guides/ieee/how-to-cite-awebsite

[4] [Wikipedia][13 May 2019] Article retrieved from URL[ https://en.wikipedia.org/wiki/Histogram_of_oriented_gradie nts

[5] [MATLAB] [13 May 2019]. Article retrieved from URL https://www.mathworks.com/help/stats/fitcecoc.html?search Highlight=fitcecoc\&s_tid=doc_srchtitle

[6] [MATLAB] [13 May 2019]. Article retrieved from URL https://www.mathworks.com/help/stats/classificationecoc.pr edict.html

[7] [MATLAB] [13 May 2019]. Article retrieved from URL www.mathworks.com 'compare strings',1994-2019. Available : $\quad$ www.mathworks.com/help/matlab/ref/ strcmp.html

[8] [www.mathworks.com 'predict, 1994-2019.]. Article retrieved from URL www.mathworks.com /help/stats/ compactclassificationdiscriminant.predict.

[9] Allwein, E., R. Schapire, and Y. Singer. "Reducing multiclass to binary: A unifying approach for margin classifiers." Journal of Machine Learning Research. Vol. 1, 2000, pp. 113-141.

[10] Escalera, S., O. Pujol, and P. Radeva. "On the decoding process in ternary error-correcting output codes." IEEE Transactions on Pattern Analysis and Machine Intelligence. Vol. 32, Issue 7, 2010, pp. 120-134.

[11] www.worldwildlife.org 'species endangered list', 2019 Available on www.worldwildlife.org/species/directory?page $=2 \&$ ssor=ext inction_status. [Accessed : 13 May 2019] 